\title{
EELS Mapping with Random Scan
}

\author{
Shixin Wang and Du Li
}

\author{
Micron Technology Inc., Boise, ID, USA
}

With modern TEM/ STEM system, elemental mapping is one of the most important components in microanalysis. The conventional mapping scheme is to raster the electron beam in a sequential fashion, from left to right and top to bottom [1]. To obtain elemental mapping, one or more detectors are used to collect signals (such as EDS and EELS) at each stopping point. The analytical signals synchronized with the electron beam position form the so called spectrum image. A typical EELS or EDS spectrum image is a 3-D numerical array with two spatial dimensions and one energy dimension.

In this work we demonstrate a randomized mapping scheme. In this random mapping, the electron beam jumps to a random-selected position within a pre-defined mapping area. When this random scanning combines with EELS or EDS signal collection, we form a spectrum image. When electron-beam stopping exhausts all the stopping points without repetition, a complete mapping cycle is completed. A multiple-cycle random mapping can be performed if necessary.

The primary advantage of random mapping is to reduce beam damage effect. For beam sensitive material, electron beam may alter the composition and/or structure of the specimen over an area large than the probe size. When this happens, the sequential mapping methods suffer significant artifacts from beam damage. The sequential stopping location is often affected by the previous beam bombardment at the spot right next to it. This may leads to cumulated beam damage effect. Many methods maybe used to reduce the beam effect, such as to lower beam intensity, to change beam energy, or using cold stage. These methods are to reduce beam damage effect based on physics. In addition to those, we can use different sampling methods, such as random mapping, to further reduce beam damage effect. One method in reducing cumulative beam damage effect with conventional raster mapping is to use large step size. But that may suffer under-sampling problem. In contrast, a random mapping jumps the beam to random positions. The probability of two sequential stopping points being next to each other is small. Within one mapping cycle, there is a large portion of data being from unaffected spots. The accumulated beam effect is reduced. Another potential advantage of random mapping is to increase recovering time of beam damaged area.

A random mapping software package is created using DigitalMicrograph $\odot$ scripting language. Fig. 1A shows the data collection interface, where scanning resolution, scanning pattern, scanning region, drift correction features, EELS parameters are set. Fig. 1B shows the reference STEM image with mapping area and drift correction area labeled by boxes. During acquisition, the electron beam jumps non-repeatedly within the area labeled by "Hopping Map" in the given mapping cycle. Fig. 1C is a snap-shot of $14 \%$ filled spectrum image from random mapping. The beam stopping position is generated by computer generated uniform random number. All beam positions are to be covered without repetition in a given mapping cycle. Sample drift is corrected at a pre-defined frequency.

Fig. 2 shows the comparison of two nitrogen maps from a specimen cross-sectioned through two SiN layers in the matrix of $\mathrm{SiO}$. The two maps are of the same spatial resolution, step size, beam condition, and dwell time. Fig.2A is the $\mathrm{N}$ map from sequential mapping and Fig. 2B is from random mapping. Clearly the $\mathrm{N}$ from random mapping gives a better defined $\mathrm{N}$ profile.

The random mapping can be used in any scanning-mode microanalysis. Random mapping can be used in conjunction with damage reducing methods. The random scanning scheme is to reduce the accumulated damage effect rather than beam damage itself.

\section{Reference:}

[1]. P. D. Nellist, in "Scanning Transmission Electron Microscopy - Imaging and Analysis", eds. by S. J. Pennycook and P. D. Nellist, (Springer, NY), p 91. 


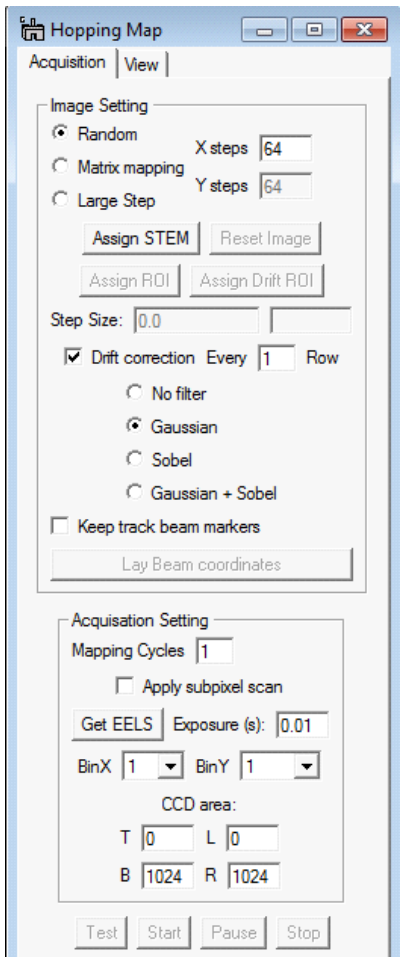

(A)

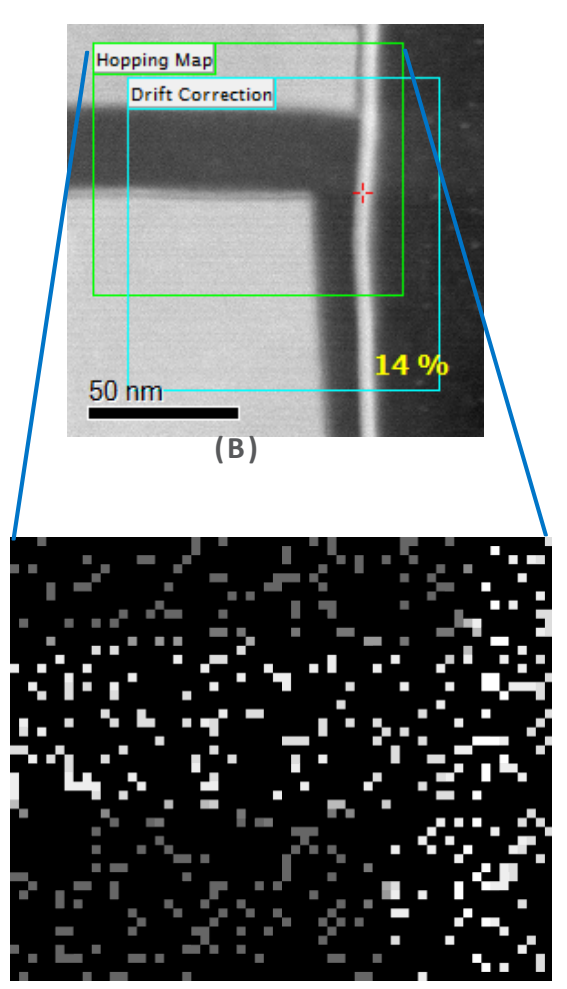

(C)

Figure 1. (A) The random mapping acquisition program interface. (B) Reference STEM image for setting up mapping area and image area used for calculating drift correction. (C) A snap-shot of partially finished spectrum image with electron beam stopped at random selected positions within the mapping area.

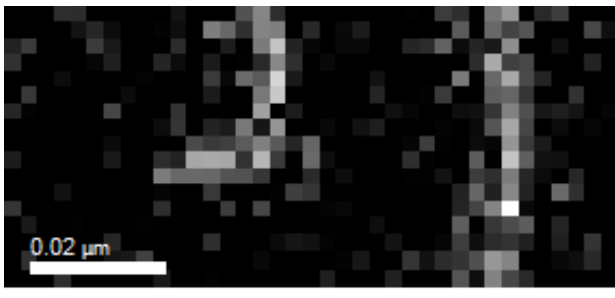

(A)

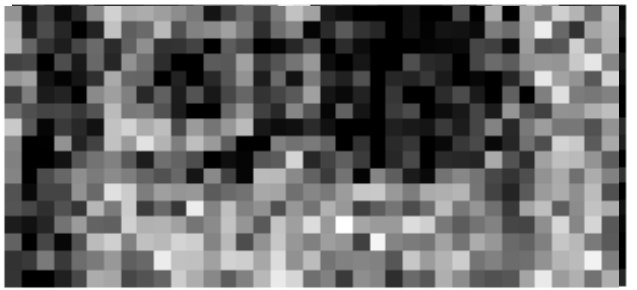

(B)

Figure 2. (A) Nitrogen map with sequential mapping. (B). Nitrogen map with random mapping. 